\title{
Fuzzy Approximate Solution of Positive Fully Fuzzy Linear Matrix Equations
}

\author{
Xiaobin Guo ${ }^{1}$ and Dequan Shang ${ }^{2}$ \\ ${ }^{1}$ College of Mathematics and Statistics, Northwest Normal University, Lanzhou 730070, China \\ ${ }^{2}$ Department of Public Courses, Gansu College of Traditional Chinese Medicine, Lanzhou 730000, China \\ Correspondence should be addressed to Dequan Shang; gxbglz@163.com
}

Received 22 November 2012; Accepted 19 January 2013

Academic Editor: Panayiotis J. Psarrakos

Copyright (c) 2013 X. Guo and D. Shang. This is an open access article distributed under the Creative Commons Attribution License, which permits unrestricted use, distribution, and reproduction in any medium, provided the original work is properly cited.

\begin{abstract}
The fuzzy matrix equations $\widetilde{A} \otimes \widetilde{X} \otimes \widetilde{B}=\widetilde{C}$ in which $\widetilde{A}, \widetilde{B}$, and $\widetilde{C}$ are $m \times m, n \times n$, and $m \times n$ nonnegative LR fuzzy numbers matrices, respectively, are investigated. The fuzzy matrix systems is extended into three crisp systems of linear matrix equations according to arithmetic operations of LR fuzzy numbers. Based on pseudoinverse of matrix, the fuzzy approximate solution of original fuzzy systems is obtained by solving the crisp linear matrix systems. In addition, the existence condition of nonnegative fuzzy solution is discussed. Two examples are calculated to illustrate the proposed method.
\end{abstract}

\section{Introduction}

Since many real-world engineering systems are too complex to be defined in precise terms, imprecision is often involved in any engineering design process. Fuzzy systems have an essential role in this fuzzy modeling, which can formulate uncertainty in actual environment. In many matrix equations, some or all of the system parameters are vague or imprecise, and fuzzy mathematics is a better tool than crisp mathematics for modeling these problems, and hence solving a fuzzy matrix equation is becoming more important. The concept of fuzzy numbers and arithmetic operations with these numbers were first introduced and investigated by Zadeh [1, 2], Dubois and Prade [3], and Nahmias [4]. A different approach to fuzzy numbers and the structure of fuzzy number spaces was given by Puri and Ralescu [5], Goetschel, Jr. and Voxman [6], and $\mathrm{Wu}$ and $\mathrm{Ma}[7,8]$.

In the past decades, many researchers have studied the fuzzy linear equations such as fuzzy linear systems (FLS), dual fuzzy linear systems (DFLS), general fuzzy linear systems (GFLS), fully fuzzy linear systems (FFLS), dual fully fuzzy linear systems (DFFLS), and general dual fuzzy linear systems (GDFLS). These works were performed mainly by Friedman et al. [9, 10], Allahviranloo et al. [11-17], Abbasbandy et al.
[18-21], Wang and Zheng [22, 23], and Dehghan et al. [24, 25]. The general method they applied is the fuzzy linear equations were converted to a crisp function system of linear equations with high order according to the embedding principles and algebraic operations of fuzzy numbers. Then the fuzzy solution of the original fuzzy linear systems was derived from solving the crisp function linear systems. However, for a fuzzy matrix equation which always has a wide use in control theory and control engineering, few works have been done in the past. In 2009, Allahviranloo et al. [26] discussed the fuzzy linear matrix equations (FLME) of the form $A \widetilde{X} B=\widetilde{C}$ in which the matrices $A$ and $B$ are known $m \times m$ and $n \times n$ real matrices, respectively; $\widetilde{C}$ is a given $m \times n$ fuzzy matrix. By using the parametric form of fuzzy number, they derived necessary and sufficient conditions for the existence condition of fuzzy solutions and designed a numerical procedure for calculating the solutions of the fuzzy matrix equations. In 2011, Guo et al. [27-29] investigated a class of fuzzy matrix equations $A \widetilde{x}=\widetilde{B}$ by means of the block Gaussian elimination method and studied the least squares solutions of the inconsistent fuzzy matrix equation $A \widetilde{x}=\widetilde{B}$ by using generalized inverses of the matrix, and discussed fuzzy symmetric solutions of fuzzy matrix equations $A \widetilde{X}=\widetilde{B}$. What is more, there are two 
shortcomings in the above fuzzy systems. The first is that in these fuzzy linear systems and fuzzy matrix systems the fuzzy elements were denoted by triangular fuzzy numbers, so the extended model equations always contain parameter $r, 0 \leq$ $r \leq 1$ which makes their computation especially numerical implementation inconvenient in some sense. The other one is that the weak fuzzy solution of fuzzy linear systems $A \tilde{x}=\widetilde{b}$ does not exist sometimes see; [30].

To make the multiplication of fuzzy numbers easy and handle the fully fuzzy systems, Dubois and Prade [3] introduced the LR fuzzy number in 1978. We know that triangular fuzzy numbers and trapezoidal fuzzy numbers [31] are just specious cases of LR fuzzy numbers. In 2006, Dehghan et al. [25] discussed firstly computational methods for fully fuzzy linear systems $\widetilde{A} \widetilde{x}=\widetilde{b}$ whose coefficient matrix and the right-hand side vector are LR fuzzy numbers. In 2012, Allahviranloo et al. studied LR fuzzy linear systems [32] by the linear programming with equality constraints. Otadi and Mosleh considered the nonnegative fuzzy solution [33] of fully fuzzy matrix equations $\widetilde{A} \widetilde{X}=\widetilde{B}$ by employing linear programming with equality constraints at the same year. Recently, Guo and Shang [34] investigated the fuzzy approximate solution of LR fuzzy Sylvester matrix equations $A \widetilde{X}+\widetilde{X} B=\widetilde{C}$. In this paper we propose a general model for solving the fuzzy linear matrix equation $\widetilde{A} \otimes \widetilde{X} \otimes \widetilde{B}=\widetilde{C}$, where $\widetilde{A}, \widetilde{B}$, and $\widetilde{C}$ are $m \times m, n \times n$, and $m \times n$ nonnegative LR fuzzy numbers matrices, respectively. The model is proposed in this way; that is, we extend the fuzzy linear matrix system into a system of linear matrix equations according to arithmetic operations of LR fuzzy numbers. The LR fuzzy solution of the original matrix equation is derived from solving crisp systems of linear matrix equations. The structure of this paper is organized as follows.

In Section 2, we recall the LR fuzzy numbers and present the concept of fully fuzzy linear matrix equation. The computing model to the positive fully fuzzy linear matrix equation is proposed in detail and the fuzzy approximate solution of the fuzzy linear matrix equation is obtained by using pseudo-inverse in Section 3. Some examples are given to illustrate our method in Section 4 and the conclusion is drawn in Section 5.

\section{Preliminaries}

\subsection{The LR Fuzzy Number}

Definition 1 (see [1]). A fuzzy number is a fuzzy set like $u$ : $R \rightarrow I=[0,1]$ which satisfies the following.

(1) $u$ is upper semicontinuous,

(2) $u$ is fuzzy convex, that is, $u(\lambda x+(1-\lambda) y) \geq$ $\min \{u(x), u(y)\}$ for all $x, y \in R, \lambda \in[0,1]$,

(3) $u$ is normal, that is, there exists $x_{0} \in R$ such that $u\left(x_{0}\right)=1$,

(4) $\operatorname{supp} u=\{x \in R \mid u(x)>0\}$ is the support of the $u$, and its closure $\mathrm{cl}(\operatorname{supp} u)$ is compact.

Let $E^{1}$ be the set of all fuzzy numbers on $R$.
Definition 2 (see [3]). A fuzzy number $\widetilde{M}$ is said to be an LR fuzzy number if

$$
\mu_{\widetilde{M}}(x)= \begin{cases}L\left(\frac{m-x}{\alpha}\right), & x \leq m, \alpha>0, \\ R\left(\frac{x-m}{\beta}\right), & x \geq m, \beta>0,\end{cases}
$$

where $m, \alpha$, and $\beta$ are called the mean value, left, and right spreads of $\widetilde{M}$, respectively. The function $L(\cdot)$, which is called left shape function satisfies

(1) $L(x)=L(-x)$,

(2) $L(0)=1$ and $L(1)=0$,

(3) $L(x)$ is nonincreasing on $[0, \infty)$.

The definition of a right shape function $R(\cdot)$ is similar to that of $L(\cdot)$.

Clearly, $\widetilde{M}=(m, \alpha, \beta)_{\mathrm{LR}}$ is positive (negative) if and only if $m-\alpha>0(m+\beta<0)$.

Also, two LR fuzzy numbers $\widetilde{M}=(m, \alpha, \beta)_{\mathrm{LR}}$ and $\widetilde{N}=$ $(n, \gamma, \delta)_{\mathrm{LR}}$ are said to be equal, if and only if $m=n, \alpha=\gamma$, and $\beta=\delta$.

Definition 3 (see [5]). For arbitrary LR fuzzy numbers $\widetilde{M}=$ $(m, \alpha, \beta)_{\mathrm{LR}}$ and $\widetilde{N}=(n, \gamma, \delta)_{\mathrm{LR}}$, we have the following.

(1) Addition:

$\widetilde{M} \oplus \widetilde{N}=(m, \alpha, \beta)_{\mathrm{LR}} \oplus(n, \gamma, \delta)_{\mathrm{LR}}=(m+n, \alpha+\gamma, \beta+\delta)_{\mathrm{LR}}$.

(2) Multiplication:

(i) If $\widetilde{M}>0$ and $\widetilde{N}>0$, then

$$
\begin{aligned}
\widetilde{M} \otimes \widetilde{N} & =(m, \alpha, \beta)_{\mathrm{LR}} \otimes(n, \gamma, \delta)_{\mathrm{LR}} \\
& \cong(m n, m \gamma+n \alpha, m \delta+n \beta)_{\mathrm{LR}}
\end{aligned}
$$

(ii) if $\widetilde{M}<0$ and $\widetilde{N}>0$, then

$$
\begin{aligned}
\widetilde{M} \otimes \widetilde{N} & =(m, \alpha, \beta)_{\mathrm{RL}} \otimes(n, \gamma, \delta)_{\mathrm{LR}} \\
& \cong(m n, n \alpha-m \delta, n \beta-m \gamma)_{\mathrm{RL}}
\end{aligned}
$$

(iii) if $\widetilde{M}<0$ and $\widetilde{N}<0$, then

$$
\begin{aligned}
\widetilde{M} \otimes \widetilde{N} & =(m, \alpha, \beta)_{\mathrm{RL}} \otimes(n, \gamma, \delta)_{\mathrm{LR}} \\
& \cong(m n,-m \delta-n \beta,-m \gamma-n \alpha)_{\mathrm{RL}},
\end{aligned}
$$

(3) Scalar multiplication:

$$
\begin{aligned}
\lambda \times \widetilde{M} & =\lambda \times(m, \alpha, \beta)_{\mathrm{LR}} \\
& = \begin{cases}(\lambda m, \lambda \alpha, \lambda \beta)_{\mathrm{LR}}, & \lambda \geq 0, \\
(\lambda m,-\lambda \beta,-\lambda \alpha)_{\mathrm{RL}}, & \lambda<0 .\end{cases}
\end{aligned}
$$




\subsection{The LR Fuzzy Matrix}

Definition 4. A matrix $\widetilde{A}=\left(\widetilde{a}_{i j}\right)$ is called an LR fuzzy matrix, if each element $\tilde{a}_{i j}$ of $\widetilde{A}$ is an LR fuzzy number.

$\widetilde{A}$ will be positive (negative) and denoted by $\widetilde{A}>0(\widetilde{A}<$ 0 ) if each element $\widetilde{a}_{i j}$ of $\widetilde{A}$ is positive (negative), where $\widetilde{a}_{i j}=$ $\left(a_{i j}, a_{i j}^{l}, a_{i j}^{r}\right)_{\mathrm{LR}}$. Up to the rest of this paper, we use positive LR fuzzy numbers and formulas given in Definition 3. For example, we represent $m \times n \operatorname{LR}$ fuzzy matrix $\widetilde{A}=\left(\widetilde{a}_{i j}\right)$, that $\tilde{a}_{i j}=\left(a_{i j}, \alpha_{i j}, \beta_{i j}\right)_{\mathrm{LR}}$ with new notation $\widetilde{A}=(A, M, N)$, where $A=\left(a_{i j}\right), M=\left(\alpha_{i j}\right)$ and $N=\left(\beta_{i j}\right)$ are three $m \times n$ crisp matrices. In particular, an $n$ dimensions LR fuzzy numbers vector $\tilde{x}$ can be denoted by $\left(x, x^{l}, x^{r}\right)$, where $x=\left(x_{i}\right), x^{l}=$ $\left(x_{i}^{l}\right)$ and $x^{r}=\left(x_{i}^{r}\right)$ are three $n$ dimensions crisp vectors.

Definition 5. Let $\widetilde{A}=\left(\widetilde{a}_{i j}\right)$ and $\widetilde{B}=\left(\widetilde{b}_{i j}\right)$ be two $m \times n$ and $n \times p$ fuzzy matrices; we define $\widetilde{A} \otimes \widetilde{B}=\widetilde{C}=\left(\widetilde{c}_{i j}\right)$ which is an $m \times p$ fuzzy matrix, where

$$
\widetilde{c}_{i j}=\sum_{k=1,2, \ldots, n}^{\oplus} \tilde{a}_{i k} \otimes \tilde{b}_{k j} .
$$

\subsection{The Fully Fuzzy Linear Matrix Equation}

Definition 6. The matrix system:

$$
\begin{aligned}
& \left(\begin{array}{cccc}
\tilde{a}_{11} & \tilde{a}_{12} & \cdots & \tilde{a}_{1 m} \\
\tilde{a}_{21} & \tilde{a}_{22} & \cdots & \tilde{a}_{2 m} \\
\vdots & \vdots & \vdots & \vdots \\
\tilde{a}_{m 1} & \tilde{a}_{m 2} & \cdots & \tilde{a}_{m m}
\end{array}\right) \otimes\left(\begin{array}{cccc}
\tilde{x}_{11} & \tilde{x}_{12} & \cdots & \tilde{x}_{1 n} \\
\tilde{x}_{21} & \tilde{x}_{22} & \cdots & \tilde{x}_{2 n} \\
\vdots & \vdots & \vdots & \vdots \\
\tilde{x}_{m 1} & \tilde{x}_{m 2} & \cdots & \tilde{x}_{m n}
\end{array}\right) \\
& \otimes\left(\begin{array}{cccc}
\tilde{b}_{11} & \tilde{b}_{12} & \cdots & \tilde{b}_{1 n} \\
\widetilde{b}_{21} & \widetilde{b}_{22} & \cdots & \widetilde{b}_{2 n} \\
\vdots & \vdots & \vdots & \vdots \\
\tilde{b}_{n 1} & \tilde{b}_{n 2} & \cdots & \tilde{b}_{n n}
\end{array}\right) \\
& =\left(\begin{array}{cccc}
\tilde{c}_{11} & \tilde{c}_{12} & \cdots & \widetilde{c}_{1 n} \\
\widetilde{c}_{21} & \widetilde{c}_{22} & \cdots & \widetilde{c}_{2 n} \\
\vdots & \vdots & \vdots & \vdots \\
\tilde{c}_{m 1} & \tilde{c}_{m 2} & \cdots & \tilde{c}_{m n}
\end{array}\right)
\end{aligned}
$$

where $\tilde{a}_{i j}, 1 \leq i, j \leq m, \widetilde{b}_{i j}, 1 \leq i, j \leq n$, and $\widetilde{c}_{i j}, 1 \leq i \leq m$, $1 \leq j \leq n$ are LR fuzzy numbers, is called an LR fully fuzzy linear matrix equation (FFLME).

Using matrix notation, we have

$$
\widetilde{A} \otimes \widetilde{X} \otimes \widetilde{B}=\widetilde{C} .
$$

A fuzzy numbers matrix

$$
\widetilde{X}=\left(x_{i j}, y_{i j}, z_{i j}\right)_{\mathrm{LR}}, \quad 1 \leq i \leq m, 1 \leq j \leq n
$$

is called an LR fuzzy approximate solution of fully fuzzy linear matrix equation (8) if $\widetilde{X}$ satisfies (9).

Up to the rest of this paper, we will discuss the nonnegative solution $\widetilde{X}=(X, Y, Z) \geq 0$ of FFLME $\widetilde{A} \otimes \widetilde{X} \otimes \widetilde{B}=\widetilde{C}$, where $\widetilde{A}=(A, M, N) \geq 0, \widetilde{B}=(B, E, F) \geq 0$, and $\widetilde{C}=$ $(C, G, H) \geq 0$.

\section{Method for Solving FFLME}

First, we extend the fuzzy linear matrix system (9) into three systems of linear matrix equations according to the LR fuzzy number and its arithmetic operations.

Theorem 7. The fuzzy linear matrix system (9) can be extended into the following model:

$$
\begin{gathered}
A X B=C, \\
A X E+A Y B+M X B=G, \\
A X F+A Z B+N X B=H .
\end{gathered}
$$

Proof. We denote $\widetilde{A}=(A, M, N), \widetilde{B}=(B, E, F)$ and $\widetilde{C}=$ $(C, G, H)$, and assume $\widetilde{X}=(X, Y, Z) \geq 0$, then

$$
\begin{aligned}
\widetilde{A} \widetilde{X} \widetilde{B} & =(A, M, N) \otimes(X, Y, Z) \otimes(B, E, F) \\
& =(A X, A Y+M X, A Z+N X) \otimes(B, E, F) \\
& =(A X B, A X E+A Y B+M X B, A X F+A Z B+N X B) \\
& =(C, G, H),
\end{aligned}
$$

according to multiplication of nonnegative LR fuzzy numbers of Definition 2. Thus we obtain a model for solving FFLME (9) as follows:

$$
\begin{gathered}
A X B=C, \\
A X E+A Y B+M X B=G, \\
A X F+A Z B+N X B=H .
\end{gathered}
$$

Secondly, in order to solve the fuzzy linear matrix equation (9), we need to consider the crisp systems of linear matrix equation (11). Supposing $A$ and $B$ are nonsingular crisp matrices, we have

$$
\begin{gathered}
X=A^{-1} C B^{-1}, \\
Y=A^{-1} G-X E B^{-1}-A^{-1} M X, \\
Z=A^{-1} H-X F B^{-1}-A^{-1} N X,
\end{gathered}
$$

that is,

$$
\begin{gathered}
X=A^{-1} C B^{-1}, \\
Y=A^{-1} G-A^{-1} C B^{-1} E B^{-1}-A^{-1} M A^{-1} C B^{-1}, \\
Z=A^{-1} H-A^{-1} C B^{-1} F B^{-1}-A^{-1} N A^{-1} C B^{-1} .
\end{gathered}
$$

Definition 8 . Let $\widetilde{X}=(X, Y, Z)$ be an LR fuzzy matrix. If $(X, Y, Z)$ is an exact solution of (11) such that $X \geq 0, Y \geq 0$, $Z \geq 0$, and $X-Y \geq 0$; we call $\widetilde{X}=(X, Y, Z)$ a nonnegative LR fuzzy approximate solution of (9). 
Theorem 9. Let $\widetilde{A}=(A, M, N), \widetilde{B}=(B, E, F)$, and $\widetilde{C}=$ $(C, G, H)$ be three nonnegative fuzzy matrices, respectively, and let $A$ and $B$ be the product of a permutation matrix by a diagonal matrix with positive diagonal entries. Moreover, let $G B \geq C B^{-1} E+M A^{-1} C, H B \geq C B^{-1} F+N A^{-1} C$, and $C+C B^{-1} E+M A^{-1} C \geq G B$. Then the systems $\widetilde{A} \otimes \widetilde{X} \otimes \widetilde{B}=\widetilde{C}$ has nonnegative fuzzy solutions.

Proof. Our hypotheses on $A$ and $B$ imply that $A^{-1}$ and $B^{-1}$ exist and they are nonnegative matrices. Thus $X=$ $A^{-1} C B^{-1} \geq 0$.

On the other hand, because $G B \geq C B^{-1} E+M A^{-1} C$ and $H B \geq C B^{-1} F+N A^{-1} C$, so with $Y=A^{-1}\left(G B-C B^{-1} E-\right.$ $\left.M A^{-1} C\right) B^{-1}$ and $Z=A^{-1}\left(H B-C B^{-1} F-N A^{-1} C\right) B^{-1}$, we have $Y \geq 0$ and $Z \geq 0$. Thus $\widetilde{X}=(X, Y, Z)$ is a fuzzy matrix which satisfies $\widetilde{A} \otimes \widetilde{X} \otimes \widetilde{B}=\widetilde{C}$. Since $X-Y=A^{-1}(C-G B+$ $\left.C B^{-1} E+M A^{-1} C\right) B^{-1}$, the positivity property of $\widetilde{X}$ can be obtained from the condition $C+C B^{-1} E+M A^{-1} C \geq G B$.

When $A$ or $B$ is a singular crisp matrix, the following result is obvious.

Theorem 10 (see [35]). For linear matrix equations $A X B=C$, where $A \in R^{m \times m}, B \in R^{n \times n}$, and $C \in R^{m \times n}$. Then

$$
X=A^{\dagger} C B^{\dagger}
$$

is its minimal norm least squares solution.

By the pseudoinverse of matrices, we solve model (11) and obtain its minimal norm least squares solution as follows:

$$
\begin{gathered}
X=A^{\dagger} C B^{\dagger}, \\
Y=A^{\dagger} G-X E B^{\dagger}-A^{\dagger} M X \\
Z=A^{\dagger} H-X F B^{\dagger}-A^{\dagger} N X,
\end{gathered}
$$

that is,

$$
\begin{gathered}
X=A^{\dagger} C B^{\dagger}, \\
Y=A^{\dagger} G-A^{\dagger} C B^{\dagger} E B^{\dagger}-A^{\dagger} M A^{\dagger} C B^{\dagger}, \\
Z=A^{\dagger} H-A^{\dagger} C B^{\dagger} F B^{\dagger}-A^{\dagger} N A^{\dagger} C B^{\dagger} .
\end{gathered}
$$

Definition 11. Let $\widetilde{X}=(X, Y, Z)$ be an LR fuzzy matrix. If $(X, Y, Z)$ is a minimal norm least squares solution of (11) such that $X \geq 0, Y \geq 0, Z \geq 0$, and $X-Y \geq 0$, we call $\widetilde{X}=(X, Y, Z)$ a nonnegative LR fuzzy minimal norm least squares solution of (9).

At last, we give a sufficient condition for nonnegative fuzzy minimal norm least squares solution of FFLME (9) in the same way.

Theorem 12. Let $A^{\dagger}$ and $B^{\dagger}$ be nonnegative matrices. Moreover, let $G B \geq C B^{\dagger} E+M A^{\dagger} C, H B \geq C B^{\dagger} F+N A^{\dagger} C$, and $C+C B^{\dagger} E+M A^{\dagger} C \geq G B$. Then the systems $\widetilde{A} \otimes \widetilde{X} \otimes \widetilde{B}=\widetilde{C}$ has nonnegative fuzzy minimal norm least squares solutions.
Proof. Since $A^{\dagger}$ and $B^{\dagger}$ are nonnegative matrices, we have $X=A^{\dagger} C B^{\dagger} \geq 0$.

Now that $G B \geq C B^{\dagger} E+M A^{\dagger} C$ and $H B \geq C B^{\dagger} F+N A^{\dagger} C$, therefore, with $Y=A^{\dagger}\left(G B-C B^{\dagger} E-M A^{\dagger} C\right) B^{\dagger}$ and $Z=$ $A^{\dagger}\left(H B-C B^{\dagger} F-N A^{\dagger} C\right) B^{\dagger}$, we have $Y \geq 0$ and $Z \geq 0$. Thus $\widetilde{X}=(X, Y, Z)$ is a fuzzy matrix which satisfies $\widetilde{A} \otimes \widetilde{X} \otimes \widetilde{B}=$ $\widetilde{C}$. Since $X-Y=A^{\dagger}\left(C-G B+C B^{\dagger} E+M A^{\dagger} C\right) B^{\dagger}$, the nonnegativity property of $\widetilde{X}$ can be obtained from the condition $C+C B^{\dagger} E+M A^{\dagger} C \geq G B$.

The following Theorems give some results for such $S^{-1}$ and $S^{\dagger}$ to be nonnegative. As usual, $(\cdot)^{\top}$ denotes the transpose of a matrix $(\cdot)$.

Theorem 13 (see [36]). The inverse of a nonnegative matrix $A$ is nonnegative if and only if $A$ is a generalized permutation matrix.

Theorem 14 (see [37]). Let $A$ be an $m \times m$ nonnegative matrix with rank $r$. Then the following assertions are equivalent:

(a) $A^{\dagger} \geq 0$.

(b) There exists a permutation matrix $P$, such that PA has the form

$$
P A=\left(\begin{array}{c}
S_{1} \\
S_{2} \\
\vdots \\
S_{r} \\
O
\end{array}\right)
$$

where each $S_{i}$ has rank 1 and the rows of $S_{i}$ are orthogonal to the rows of $S_{j}$, whenever $i \neq j$, the zero matrix may be absent.

(c) $A^{\dagger}=\left(\begin{array}{ll}K P^{\top} & K Q^{\top} \\ K P^{\top} & K P^{\top}\end{array}\right)$ for some positive diagonal matrix $K$. In this case,

$$
(P+Q)^{\dagger}=K(P+Q)^{\top}, \quad(P-Q)^{\dagger}=K(P-Q)^{\top} .
$$

\section{Numerical Examples}

Example 15. Consider the fully fuzzy linear matrix system

$$
\begin{gathered}
\left(\begin{array}{ll}
(2,1,1)_{\mathrm{LR}} & (1,0,1)_{\mathrm{LR}} \\
(1,0,0)_{\mathrm{LR}} & (2,1,0)_{\mathrm{LR}}
\end{array}\right) \otimes\left(\begin{array}{lll}
\tilde{x}_{11} & \tilde{x}_{12} & \tilde{x}_{13} \\
\tilde{x}_{21} & \tilde{x}_{22} & \tilde{x}_{23}
\end{array}\right) \\
\otimes\left(\begin{array}{lll}
(1,0,1)_{\mathrm{LR}} & (2,1,1)_{\mathrm{LR}} & (1,1,0)_{\mathrm{LR}} \\
(2,1,0)_{\mathrm{LR}} & (1,0,0)_{\mathrm{LR}} & (2,1,1)_{\mathrm{LR}} \\
(1,0,0)_{\mathrm{LR}} & (2,1,1)_{\mathrm{LR}} & (1,0,1)_{\mathrm{LR}}
\end{array}\right) \\
=\left(\begin{array}{lll}
(17,12,23)_{\mathrm{LR}} & (22,22,29)_{\mathrm{LR}} & (17,16,28)_{\mathrm{LR}} \\
(19,15,13)_{\mathrm{LR}} & (23,23,16)_{\mathrm{LR}} & (19,16,17)_{\mathrm{LR}}
\end{array}\right) .
\end{gathered}
$$


By Theorem 7, the model of the above fuzzy linear matrix system is made of the following three crisp systems of linear matrix equations

$$
\begin{aligned}
& \left(\begin{array}{ll}
2 & 1 \\
1 & 2
\end{array}\right)\left(\begin{array}{lll}
x_{11} & x_{12} & x_{13} \\
x_{21} & x_{22} & x_{23}
\end{array}\right)\left(\begin{array}{lll}
1 & 2 & 1 \\
2 & 1 & 2 \\
1 & 2 & 1
\end{array}\right)=\left(\begin{array}{lll}
17 & 22 & 17 \\
19 & 23 & 19
\end{array}\right), \\
& \left(\begin{array}{ll}
2 & 1 \\
1 & 2
\end{array}\right)\left(\begin{array}{lll}
x_{11} & x_{12} & x_{13} \\
x_{21} & x_{22} & x_{23}
\end{array}\right)\left(\begin{array}{lll}
0 & 1 & 1 \\
1 & 0 & 1 \\
0 & 1 & 0
\end{array}\right) \\
& +\left(\begin{array}{ll}
2 & 1 \\
1 & 2
\end{array}\right)\left(\begin{array}{lll}
y_{11} & y_{12} & y_{13} \\
y_{21} & y_{22} & y_{23}
\end{array}\right)\left(\begin{array}{lll}
1 & 2 & 1 \\
2 & 1 & 2 \\
1 & 2 & 1
\end{array}\right) \\
& +\left(\begin{array}{ll}
1 & 0 \\
0 & 1
\end{array}\right)\left(\begin{array}{lll}
x_{11} & x_{12} & x_{13} \\
x_{21} & x_{22} & x_{23}
\end{array}\right)\left(\begin{array}{lll}
1 & 2 & 1 \\
2 & 1 & 2 \\
1 & 2 & 1
\end{array}\right)=\left(\begin{array}{lll}
12 & 22 & 16 \\
15 & 23 & 20
\end{array}\right), \\
& \left(\begin{array}{ll}
2 & 1 \\
1 & 2
\end{array}\right)\left(\begin{array}{lll}
x_{11} & x_{12} & x_{13} \\
x_{21} & x_{22} & x_{23}
\end{array}\right)\left(\begin{array}{lll}
1 & 1 & 0 \\
0 & 0 & 1 \\
0 & 1 & 1
\end{array}\right) \\
& +\left(\begin{array}{ll}
2 & 1 \\
1 & 2
\end{array}\right)\left(\begin{array}{lll}
z_{11} & z_{12} & z_{13} \\
z_{21} & z_{22} & z_{23}
\end{array}\right)\left(\begin{array}{lll}
1 & 2 & 1 \\
2 & 1 & 2 \\
1 & 2 & 1
\end{array}\right) \\
& +\left(\begin{array}{ll}
1 & 1 \\
0 & 0
\end{array}\right)\left(\begin{array}{lll}
x_{11} & x_{12} & x_{13} \\
x_{21} & x_{22} & x_{23}
\end{array}\right)\left(\begin{array}{lll}
1 & 2 & 1 \\
2 & 1 & 2 \\
1 & 2 & 1
\end{array}\right)=\left(\begin{array}{lll}
23 & 29 & 28 \\
13 & 16 & 17
\end{array}\right) .
\end{aligned}
$$

Now that the matrix $B$ is singular, according to formula (18), the solutions of the above three systems of linear matrix equations are as follows:

$$
\begin{aligned}
X= & A^{\dagger} C B^{\dagger}=\left(\begin{array}{ll}
2 & 1 \\
1 & 2
\end{array}\right)^{\dagger}\left(\begin{array}{lll}
17 & 22 & 17 \\
19 & 23 & 19
\end{array}\right)\left(\begin{array}{lll}
1 & 2 & 1 \\
2 & 1 & 2 \\
1 & 2 & 1
\end{array}\right)^{\dagger} \\
= & \left(\begin{array}{lll}
1.5000 & 1.0000 & 1.5000 \\
1.5000 & 2.0000 & 1.5000
\end{array}\right), \\
Y= & A^{\dagger} G-X E B^{\dagger}-A^{\dagger} M X=\left(\begin{array}{ll}
2 & 1 \\
1 & 2
\end{array}\right)^{\dagger}\left(\begin{array}{lll}
12 & 22 & 16 \\
19 & 23 & 19
\end{array}\right) \\
& -X\left(\begin{array}{lll}
0 & 1 & 1 \\
1 & 0 & 1 \\
0 & 1 & 0
\end{array}\right)\left(\begin{array}{lll}
1 & 2 & 1 \\
2 & 1 & 2 \\
1 & 2 & 1
\end{array}\right)^{\dagger}-\left(\begin{array}{ll}
2 & 1 \\
1 & 2
\end{array}\right)^{\dagger}\left(\begin{array}{ll}
1 & 0 \\
0 & 1
\end{array}\right) X \\
= & \left(\begin{array}{lll}
1.0333 & 0.6667 & 0.8725 \\
1.1250 & 1.6553 & 1.2578
\end{array}\right), \\
Z= & A^{\dagger} H-X F B^{\dagger}-A^{\dagger} N X=\left(\begin{array}{ll}
2 & 1 \\
1 & 2
\end{array}\right)^{\dagger}\left(\begin{array}{lll}
23 & 29 & 28 \\
13 & 16 & 17
\end{array}\right) \\
& -X\left(\begin{array}{lll}
1 & 1 & 0 \\
0 & 0 & 1 \\
0 & 1 & 1
\end{array}\right)\left(\begin{array}{lll}
1 & 2 & 1 \\
2 & 1 & 2 \\
1 & 2 & 1
\end{array}\right)-\left(\begin{array}{ll}
2 & 1 \\
1 & 2
\end{array}\right)^{\dagger}\left(\begin{array}{ll}
1 & 1 \\
0 & 0
\end{array}\right) X \\
= & \left(\begin{array}{ccc}
8.3333 & 11.6667 & 9.3333 \\
1.4167 & 1.3333 & 2.4167
\end{array}\right) .
\end{aligned}
$$

By Definition 11, we know that the original fuzzy linear matrix equations have a nonnegative LR fuzzy solution

$$
\widetilde{X}=\left(\begin{array}{lll}
\widetilde{x}_{11} & \widetilde{x}_{12} & \widetilde{x}_{13} \\
\widetilde{x}_{21} & \widetilde{x}_{22} & \widetilde{x}_{23}
\end{array}\right)=\left(\begin{array}{ccc}
(1.5000,1.0333,8.3333)_{\mathrm{LR}} & (1.0000,0.6667,11.6667)_{\mathrm{LR}} & (1.5000,0.8725,9.3333)_{\mathrm{LR}} \\
(1.5000,1.1250,1.4167)_{\mathrm{LR}} & (2.0000,1.6553,1.3333)_{\mathrm{LR}} & (1.5000,1.2578,2.4167)_{\mathrm{LR}}
\end{array}\right),
$$

since $X \geq 0, Y \geq 0, Z \geq 0$, and $X-Y \geq 0$.

Example 16. Consider the following fuzzy matrix system:

$$
\begin{gathered}
\left(\begin{array}{ll}
(1,1,0)_{\mathrm{LR}} & (2,0,1)_{\mathrm{LR}} \\
(2,0,1)_{\mathrm{LR}} & (1,0,0)_{\mathrm{LR}}
\end{array}\right)\left(\begin{array}{ll}
\tilde{x}_{11} & \tilde{x}_{12} \\
\tilde{x}_{21} & \widetilde{x}_{22}
\end{array}\right)\left(\begin{array}{ll}
(1,0,1)_{\mathrm{LR}} & (2,1,0)_{\mathrm{LR}} \\
(2,1,1)_{\mathrm{LR}} & (3,1,2)_{\mathrm{LR}}
\end{array}\right) \\
=\left(\begin{array}{ll}
(15,14,18)_{\mathrm{LR}} & (25,24,24)_{\mathrm{LR}} \\
(12,10,12)_{\mathrm{LR}} & (20,17,15)_{\mathrm{LR}}
\end{array}\right) .
\end{gathered}
$$

By Theorem 7, the model of the above fuzzy linear matrix system is made of following three crisp systems of linear matrix equations:

$$
\begin{aligned}
& \left(\begin{array}{ll}
2 & 1 \\
1 & 2
\end{array}\right)\left(\begin{array}{ll}
x_{11} & x_{12} \\
x_{21} & x_{22}
\end{array}\right)\left(\begin{array}{ll}
1 & 2 \\
2 & 3
\end{array}\right)=\left(\begin{array}{ll}
15 & 25 \\
10 & 20
\end{array}\right), \\
& \left(\begin{array}{ll}
2 & 1 \\
1 & 2
\end{array}\right)\left(\begin{array}{ll}
x_{11} & x_{12} \\
x_{21} & x_{22}
\end{array}\right)\left(\begin{array}{ll}
0 & 1 \\
1 & 1
\end{array}\right)+\left(\begin{array}{ll}
2 & 1 \\
1 & 2
\end{array}\right)\left(\begin{array}{ll}
y_{11} & y_{12} \\
y_{21} & y_{22}
\end{array}\right)\left(\begin{array}{ll}
1 & 2 \\
2 & 3
\end{array}\right) \\
& +\left(\begin{array}{ll}
1 & 0 \\
0 & 0
\end{array}\right)\left(\begin{array}{ll}
x_{11} & x_{12} \\
x_{21} & x_{22}
\end{array}\right)\left(\begin{array}{ll}
1 & 2 \\
2 & 3
\end{array}\right)=\left(\begin{array}{ll}
14 & 24 \\
10 & 17
\end{array}\right), \\
& \left(\begin{array}{ll}
2 & 1 \\
1 & 2
\end{array}\right)\left(\begin{array}{ll}
x_{11} & x_{12} \\
x_{21} & x_{22}
\end{array}\right)\left(\begin{array}{ll}
1 & 0 \\
1 & 2
\end{array}\right)+\left(\begin{array}{ll}
2 & 1 \\
1 & 2
\end{array}\right)\left(\begin{array}{ll}
z_{11} & z_{12} \\
z_{21} & z_{22}
\end{array}\right)\left(\begin{array}{ll}
1 & 2 \\
2 & 3
\end{array}\right) \\
& +\left(\begin{array}{ll}
0 & 1 \\
1 & 0
\end{array}\right)\left(\begin{array}{ll}
x_{11} & x_{12} \\
x_{21} & x_{22}
\end{array}\right)\left(\begin{array}{ll}
1 & 2 \\
2 & 3
\end{array}\right)=\left(\begin{array}{ll}
18 & 24 \\
12 & 15
\end{array}\right) .
\end{aligned}
$$


By the same way, we obtain that the solutions of the above three systems of linear matrix equations are as follows:

$$
\begin{gathered}
X=A^{-1} C B^{-1}=\left(\begin{array}{ll}
1 & 1 \\
2 & 2
\end{array}\right), \\
Y=A^{-1} G-X E B^{-1}-A^{-1} M X=\left(\begin{array}{ll}
0 & 1 \\
0 & 1
\end{array}\right), \\
Z=A^{-1} H-X F B^{-1}-A^{-1} N X=\left(\begin{array}{ll}
0 & 0 \\
1 & 0
\end{array}\right) .
\end{gathered}
$$

Since $X \geq 0, Y \geq 0, Z \geq 0$, and $X-Y \geq 0$, we know that the original fuzzy linear matrix equations have a nonnegative LR fuzzy solution given by

$$
\begin{aligned}
\widetilde{X} & =\left(\begin{array}{ll}
\tilde{x}_{11} & \tilde{x}_{12} \\
\tilde{x}_{21} & \tilde{x}_{22}
\end{array}\right) \\
& =\left(\begin{array}{ll}
(1.000,1.000,0.000)_{\mathrm{LR}} & (1.000,1.000,0.000)_{\mathrm{LR}} \\
(2.000,0.000,1.000)_{\mathrm{LR}} & (2.000,1.000,0.000)_{\mathrm{LR}}
\end{array}\right) .
\end{aligned}
$$

\section{Conclusion}

In this work we presented a model for solving fuzzy linear matrix equations $\widetilde{A} \otimes \widetilde{X} \otimes \widetilde{B}=\widetilde{C}$ in which $\widetilde{A}$ and $\widetilde{B}$ are $m \times m$ and $n \times n$ fuzzy matrices, respectively, and $\widetilde{C}$ is an $m \times n$ arbitrary LR fuzzy numbers matrix. The model was made of three crisp systems of linear equations which determined the mean value and the left and right spreads of the solution. The LR fuzzy approximate solution of the fuzzy linear matrix equation was derived from solving the crisp systems of linear matrix equations. In addition, the existence condition of strong LR fuzzy solution was studied. Numerical examples showed that our method is feasible to solve this type of fuzzy matrix equations. Based on LR fuzzy numbers and their operations, we can investigate all kinds of fully fuzzy matrix equations in future.

\section{Acknowledgments}

The work is supported by the Natural Scientific Funds of China (no. 71061013) and the Youth Research Ability Project of Northwest Normal University (NWNU-LKQN-1120).

\section{References}

[1] L. A. Zadeh, "Fuzzy sets," Information and Computation, vol. 8, pp. 338-353, 1965.

[2] L. A. Zadeh, "The concept of a linguistic variable and its application to approximate reasoning. I," Information Science, vol. 8, pp. 199-249, 1975.

[3] D. Dubois and H. Prade, "Operations on fuzzy numbers," International Journal of Systems Science, vol. 9, no. 6, pp. 613$626,1978$.

[4] S. Nahmias, "Fuzzy variables," Fuzzy Sets and Systems. An International Journal in Information Science and Engineering, vol. 1, no. 2, pp. 97-110, 1978.
[5] M. L. Puri and D. A. Ralescu, "Differentials of fuzzy functions," Journal of Mathematical Analysis and Applications, vol. 91, no. 2, pp. 552-558, 1983.

[6] R. Goetschel, Jr. and W. Voxman, "Elementary fuzzy calculus," Fuzzy Sets and Systems, vol. 18, no. 1, pp. 31-43, 1986.

[7] C. X. Wu and M. Ma, "Embedding problem of fuzzy number space. I," Fuzzy Sets and Systems, vol. 44, no. 1, pp. 33-38, 1991.

[8] C. X. Wu and M. Ma, "Embedding problem of fuzzy number space. III," Fuzzy Sets and Systems, vol. 46, no. 2, pp. 281-286, 1992.

[9] M. Friedman, M. Ming, and A. Kandel, "Fuzzy linear systems," Fuzzy Sets and Systems, vol. 96, no. 2, pp. 201-209, 1998.

[10] M. Ma, M. Friedman, and A. Kandel, "Duality in fuzzy linear systems," Fuzzy Sets and Systems, vol. 109, no. 1, pp. 55-58, 2000.

[11] T. Allahviranloo and M. Ghanbari, "On the algebraic solution of fuzzy linear systems based on interval theory," Applied Mathematical Modelling, vol. 36, no. 11, pp. 5360-5379, 2012.

[12] T. Allahviranloo and S. Salahshour, "Fuzzy symmetric solutions of fuzzy linear systems," Journal of Computational and Applied Mathematics, vol. 235, no. 16, pp. 4545-4553, 2011.

[13] T. Allahviranloo and M. Ghanbari, "A new approach to obtain algebraic solution of interval linear systems," Soft Computing, vol. 16, no. 1, pp. 121-133, 2012.

[14] T. Allahviranloo, "Numerical methods for fuzzy system of linear equations," Applied Mathematics and Computation, vol. 155, no. 2, pp. 493-502, 2004.

[15] T. Allahviranloo, "Successive over relaxation iterative method for fuzzy system of linear equations," Applied Mathematics and Computation, vol. 162, no. 1, pp. 189-196, 2005.

[16] T. Allahviranloo, "The Adomian decomposition method for fuzzy system of linear equations," Applied Mathematics and Computation, vol. 163, no. 2, pp. 553-563, 2005.

[17] R. Nuraei, T. Allahviranloo, and M. Ghanbari, "Finding an inner estimation of the so- lution set of a fuzzy linear system," Applied Mathematical Modelling, vol. 37, no. 7, pp. 5148-5161, 2013.

[18] S. Abbasbandy, R. Ezzati, and A. Jafarian, "LU decomposition method for solving fuzzy system of linear equations," Applied Mathematics and Computation, vol. 172, no. 1, pp. 633-643, 2006.

[19] S. Abbasbandy, A. Jafarian, and R. Ezzati, "Conjugate gradient method for fuzzy symmetric positive definite system of linear equations," Applied Mathematics and Computation, vol. 171, no. 2, pp. 1184-1191, 2005.

[20] S. Abbasbandy, M. Otadi, and M. Mosleh, "Minimal solution of general dual fuzzy linear systems," Chaos, Solitons \& Fractals, vol. 37, no. 4, pp. 1113-1124, 2008.

[21] B. Asady, S. Abbasbandy, and M. Alavi, "Fuzzy general linear systems," Applied Mathematics and Computation, vol. 169, no. 1, pp. 34-40, 2005.

[22] K. Wang and B. Zheng, "Inconsistent fuzzy linear systems," Applied Mathematics and Computation, vol. 181, no. 2, pp. 973981, 2006.

[23] B. Zheng and K. Wang, "General fuzzy linear systems," Applied Mathematics and Computation, vol. 181, no. 2, pp. 1276-1286, 2006.

[24] M. Dehghan and B. Hashemi, "Iterative solution of fuzzy linear systems," Applied Mathematics and Computation, vol. 175, no. 1, pp. 645-674, 2006.

[25] M. Dehghan, B. Hashemi, and M. Ghatee, "Solution of the fully fuzzy linear systems using iterative techniques," Chaos, Solitons \& Fractals, vol. 34, no. 2, pp. 316-336, 2007. 
[26] T. Allahviranloo, N. Mikaeilvand, and M. Barkhordary, "Fuzzy linear matrix equation," Fuzzy Optimization and Decision Making, vol. 8, no. 2, pp. 165-177, 2009.

[27] X. Guo and Z. Gong, "Block Gaussian elimination methods for fuzzy matrix equations," International Journal of Pure and Applied Mathematics, vol. 58, no. 2, pp. 157-168, 2010.

[28] Z. Gong and X. Guo, "Inconsistent fuzzy matrix equations and its fuzzy least squares solutions," Applied Mathematical Modelling, vol. 35, no. 3, pp. 1456-1469, 2011.

[29] X. Guo and D. Shang, "Fuzzy symmetric solutions of fuzzy matrix equations," Advances in Fuzzy Systems, vol. 2012, Article ID 318069, 9 pages, 2012.

[30] T. Allahviranloo, M. Ghanbari, A. A. Hosseinzadeh, E. Haghi, and R. Nuraei, "A note on 'Fuzzy linear systems", Fuzzy Sets and Systems, vol. 177, pp. 87-92, 2011.

[31] C.-T. Yeh, "Weighted semi-trapezoidal approximations of fuzzy numbers," Fuzzy Sets and Systems, vol. 165, pp. 61-80, 2011.

[32] T. Allahviranloo, F. H. Lotfi, M. K. Kiasari, and M. Khezerloo, "On the fuzzy solution of LR fuzzy linear systems," Applied Mathematical Modelling, vol. 37, no. 3, pp. 1170-1176, 2013.

[33] M. Otadi and M. Mosleh, "Solving fully fuzzy matrix equations," Applied Mathematical Modelling, vol. 36, no. 12, pp. 6114-6121, 2012.

[34] X. B. Guo and D. Q. Shang, "Approximate solutions of LR fuzzy Sylvester matrix equations," Journal of Applied Mathematics. In press.

[35] A. Ben-Israel and T. N. E. Greville, Generalized Inverses: Theory and Applications, Springer-Verlag, New York, NY, USA, 2nd edition, 2003.

[36] A. Berman and R. J. Plemmons, Nonnegative Matrices in the Mathematical Sciences, Academic Press, New York, NY, USA, 1979.

[37] R. J. Plemmons, "Regular nonnegative matrices," Proceedings of the American Mathematical Society, vol. 39, pp. 26-32, 1973. 


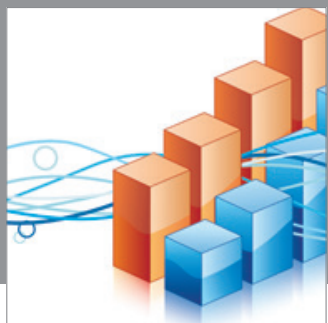

Advances in

Operations Research

mansans

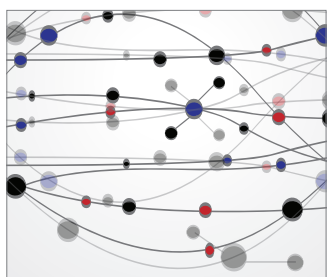

The Scientific World Journal
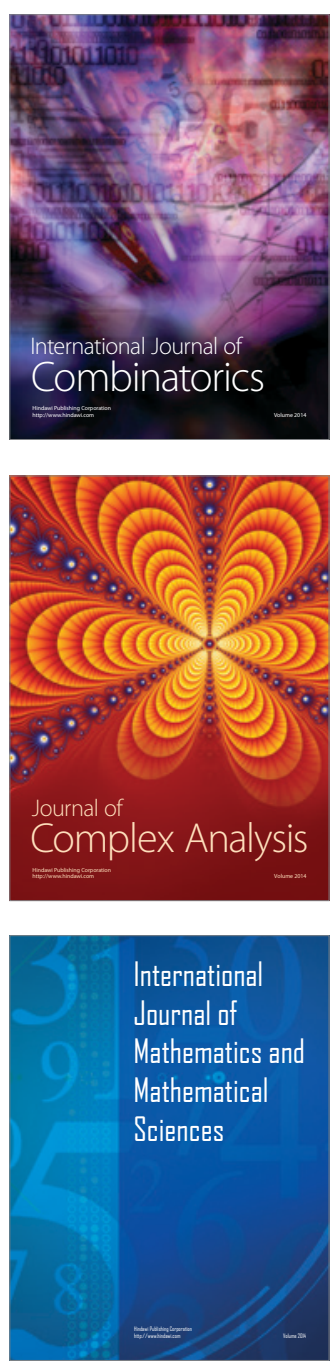
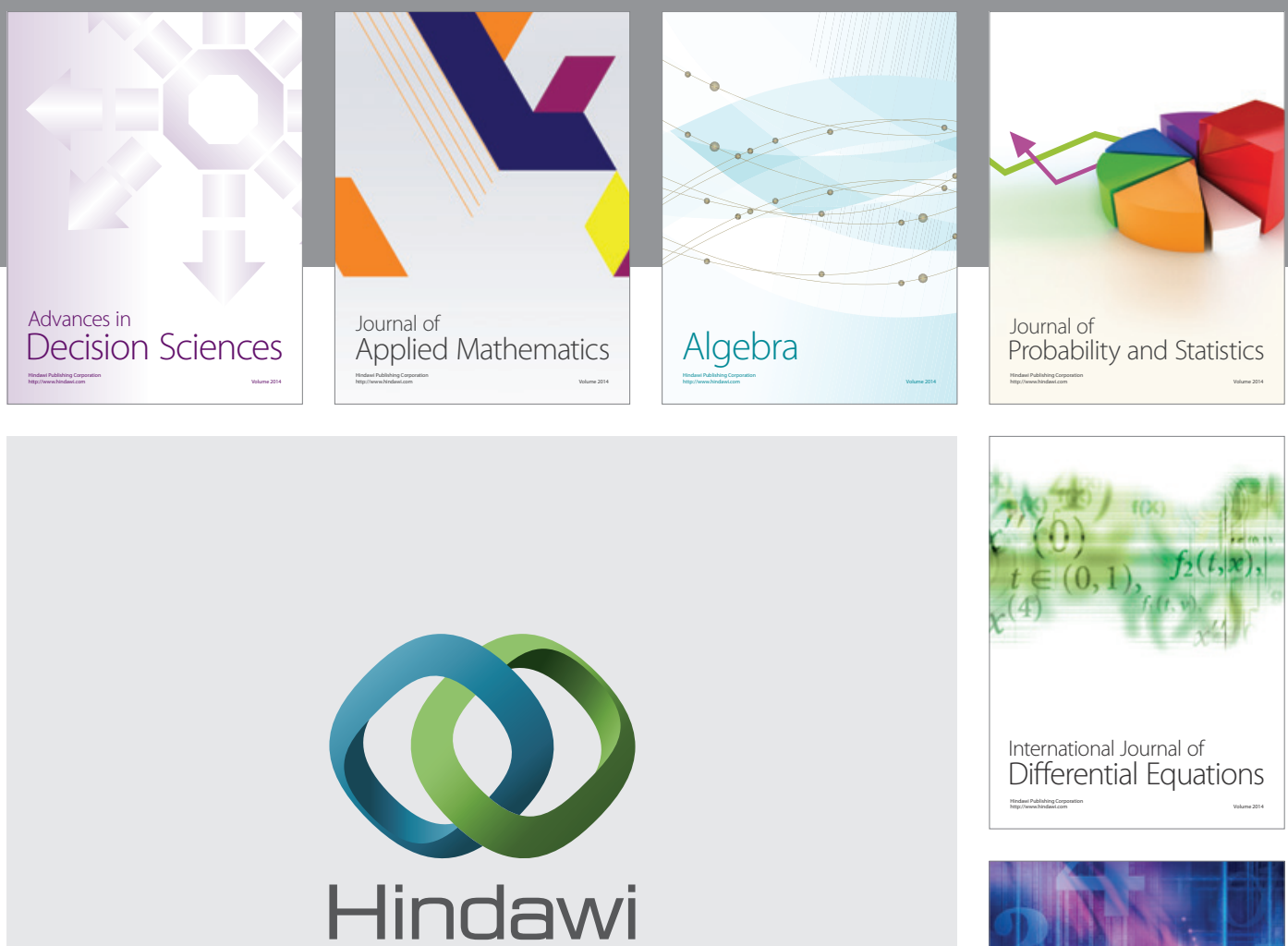

Submit your manuscripts at http://www.hindawi.com
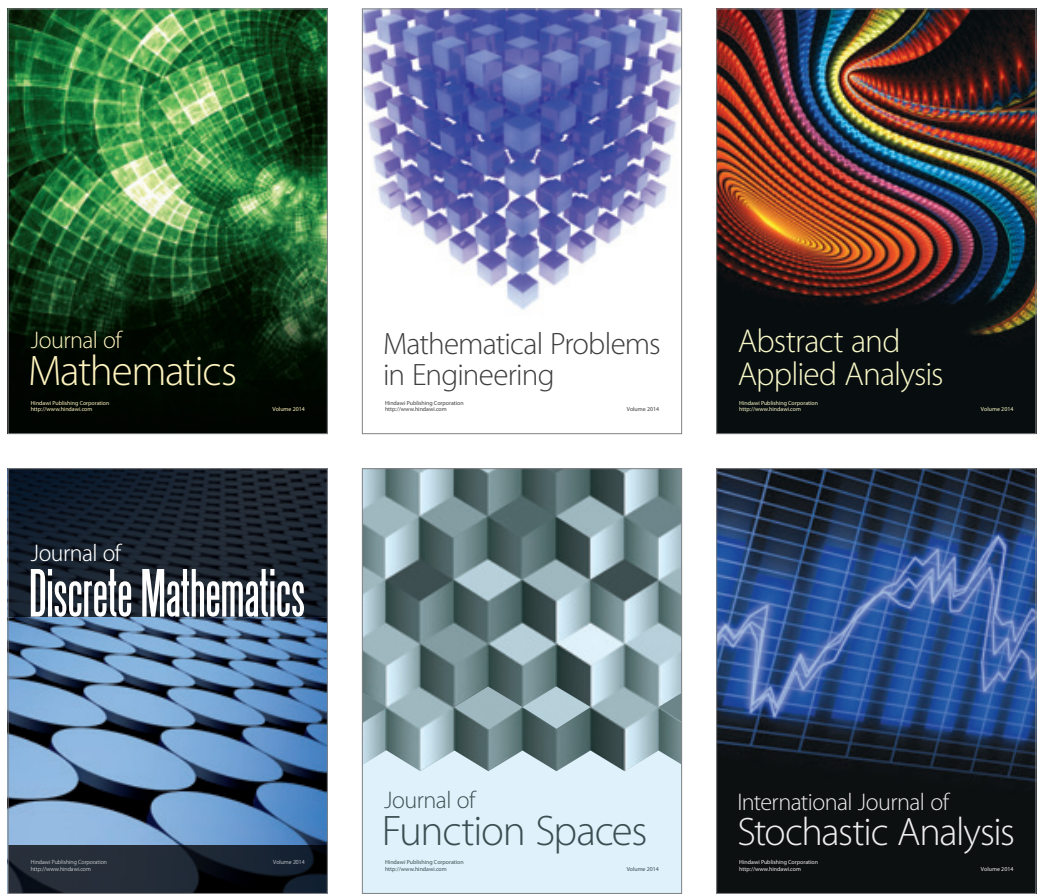

Journal of

Function Spaces

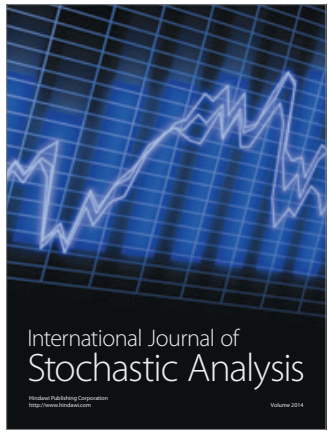

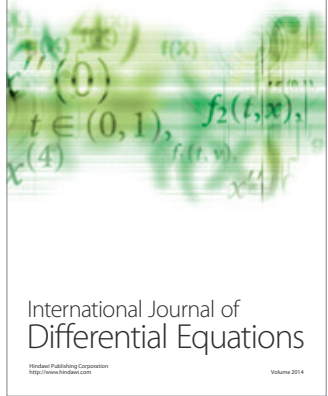
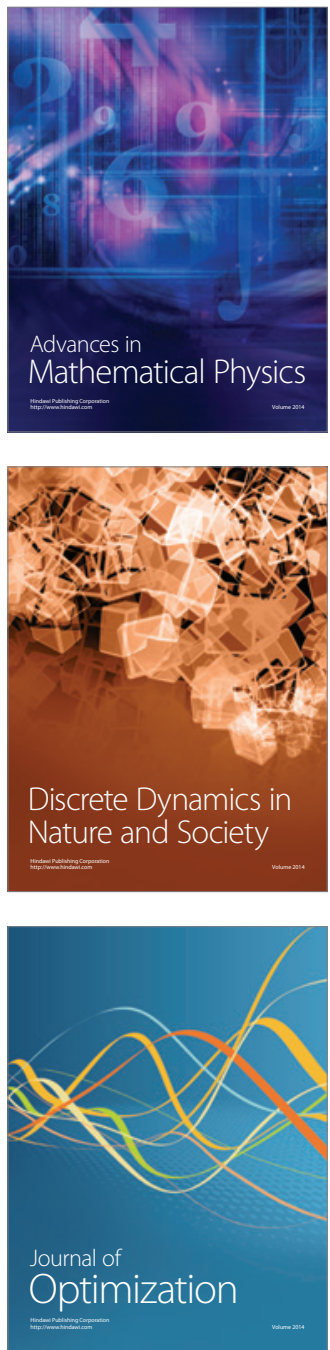